\title{
Nonperturbative Renormalization of Nonlocal Quark Bilinears for Parton Quasidistribution Functions on the Lattice Using an Auxiliary Field
}

\author{
Jeremy Green, Karl Jansen, and Fernanda Steffens \\ NIC, Deutsches Elektronen-Synchrotron, 15738 Zeuthen, Germany
}

(Received 2 August 2017; revised manuscript received 22 January 2018; published 13 July 2018)

\begin{abstract}
Parton quasidistribution functions provide a path toward an $a b$ initio calculation of parton distribution functions (PDFs) using lattice QCD. One of the problems faced in calculations of quasi-PDFs is the renormalization of a nonlocal operator. By introducing an auxiliary field, we can replace the nonlocal operator with a pair of local operators in an extended theory. On the lattice, this is closely related to the static quark theory. In this approach, we show how to understand the pattern of mixing that is allowed by chiral symmetry breaking and obtain a master formula for renormalizing the nonlocal operator that depends on three parameters. We present an approach for nonperturbatively determining these parameters and use perturbation theory to convert to the modified minimal subtraction $(\overline{\mathrm{MS}})$ scheme. Renormalization parameters are obtained for two lattice spacings using Wilson twisted mass fermions and for different discretizations of the Wilson line in the nonlocal operator. Using these parameters, we show the effect of renormalization on nucleon matrix elements with a pion mass of approximately $370 \mathrm{MeV}$ and compare renormalized results for the two lattice spacings. The renormalized matrix elements are consistent among the different Wilson line discretizations and lattice spacings.
\end{abstract}

DOI: 10.1103/PhysRevLett.121.022004

Parton distribution functions (PDFs) describe the distribution of quarks and gluons inside a proton with respect to its longitudinal momentum. They are universal: the same PDFs appear in many different scattering processes, and they are phenomenologically determined from global fits to collider data. Except for their lowest Mellin moments, PDFs have resisted $a b$ initio calculation. Lattice QCD can be used to calculate many properties of protons, but it is an inherently Euclidean space method, whereas PDFs are defined in Minkowski space via the matrix elements of operators with quark creation and annihilation separated along the light cone (here we focus on quark and antiquark PDFs rather than gluon PDFs). A possible solution to this problem was proposed by Ji [1]: compute quasi-PDFs using matrix elements of equal-time operators

$$
\mathcal{O}_{\Gamma}(x, \xi, n) \equiv \bar{\psi}(x+\xi n) \Gamma W(x+\xi n, x) \psi(x),
$$

where the $\psi$ and $\bar{\psi}$ are spatially separated by distance $\xi$ in direction $n$ and connected by a straight Wilson line $W$. From quasi-PDFs, we can obtain PDFs via a matching formula $[2,3]$, in the limit where the proton's momentum component $p \cdot n$ goes to infinity.

Published by the American Physical Society under the terms of the Creative Commons Attribution 4.0 International license. Further distribution of this work must maintain attribution to the author(s) and the published article's title, journal citation, and DOI. Funded by SCOAP.
One of the challenges in lattice calculations of quasi-PDFs is the renormalization of $\mathcal{O}_{\Gamma}$, which is a nonlocal operator and is known to be power-law divergent [4,5]. This operator also appears in the related approach of pseudo-PDFs [6,7], and similar nonlocal operators are used to study transverse momentum-dependent PDFs [8-10]. The initial lattice studies of quasi-PDFs [3,11-13] did not include a complete renormalization, but they did build the Wilson line using smeared gauge links, which has been shown in perturbation theory to reduce the power divergence [4].

Renormalization of $\mathcal{O}_{\Gamma}$ was studied in one-loop lattice perturbation theory in Ref. [14], where it was found that chiral symmetry breaking allows $\mathcal{O}_{\Gamma}$ to mix with $\mathcal{O}_{\{h, \Gamma\}}$. Numerical evidence for this mixing was also found in Ref. [10]. The study of nonperturbative renormalization was pioneered in Refs. [15,16], which used the Rome-Southampton method [17] to obtain a complex $\xi$-dependent renormalization factor (or matrix, when there is mixing) $Z(\xi)$ in the regularization independent momentum-subtraction (RI-MOM) scheme. This was then supplemented by a perturbative conversion [14] to the modified minimal subtraction $(\overline{\mathrm{MS}})$ scheme used in phenomenology. The problems with this method are that a whole function, rather than a handful of parameters, must be determined and that conversion at large $\xi$ may occur outside the regime where perturbation theory is valid. Since the intermediate scheme fixes more than the minimal number of parameters, this means that nonperturbative information in correlation functions is lost, only to be recovered perturbatively in the conversion to $\overline{\mathrm{MS}}$. 
In this work, we study the use of an auxiliary scalar, color triplet field $\zeta(\xi)$ defined only on the line $x+\xi n$ to simplify the renormalization of $\mathcal{O}_{\Gamma}$. In this approach, we replace correlation functions in QCD involving $\mathcal{O}_{\Gamma}$ with correlation functions in the extended theory $\mathrm{QCD}+\zeta$ involving the local color singlet bilinear $\phi \equiv \bar{\zeta} \psi$. This approach has been used long ago in the continuum $[18,19]$.

On the lattice, for now we restrict $n$ to point along one of the axes, $n= \pm \hat{\mu}$, and use the action

$$
\begin{aligned}
& S_{\zeta}=a \sum_{\xi} \frac{1}{1+a m_{0}} \bar{\zeta}(x+\xi n)\left[\nabla_{n}+m_{0}\right] \zeta(x+\xi n), \\
& \nabla_{n} \equiv \begin{cases}n \cdot \nabla^{*}=\nabla_{\mu}^{*} & \text { if } n=+\hat{\mu}, \\
n \cdot \nabla=-\nabla_{\mu} & \text { if } n=-\hat{\mu},\end{cases}
\end{aligned}
$$

where $\nabla$ and $\nabla^{*}$ are the forward and backward lattice covariant derivatives, respectively, and $a$ is the lattice spacing. This yields the bare propagator in a fixed gauge background:

$$
\langle\zeta(x+\xi n) \bar{\zeta}(x)\rangle_{\zeta}=\theta(\xi) e^{-m \xi} W(x+\xi n, x),
$$

where $m=a^{-1} \log \left(1+a m_{0}\right)$ and $W$ is the simple product of lattice gauge links connecting $x$ and $x+\xi n$. Smeared gauge links can be used to construct $W$ by using the same gauge links to define $\nabla_{n}$. The mass term cannot be forbidden by any symmetry and corresponds to an $O\left(a^{-1}\right)$ counterterm. Using this propagator, we obtain for $m=0$ and $\xi>0$ the relation

$$
\mathcal{O}_{\Gamma}(x, \xi, n)=\langle\bar{\phi}(x+\xi n) \Gamma \phi(x)\rangle_{\zeta} .
$$

Thus, the auxiliary field allows us to rewrite the bare nonlocal operator as the product of two local composite operators, such that standard renormalization prescriptions can be applied. To obtain $\mathcal{O}_{\Gamma}$ for $\xi<0$, we reverse the direction in which $\zeta$ propagates and use $\mathcal{O}_{\Gamma}(x, \xi, n)=$ $\mathcal{O}_{\Gamma}(x,-\xi,-n)$.

In addition to determining the counterterm $m_{0}$, we must also renormalize the local composite operator $\phi$. This can mix with any operator of the same or lower dimension, provided that they transform in the same way under all symmetries of the action. Noting that $n$ is a special direction in $S_{\zeta}$ that partially breaks rotational symmetry, we find that $\phi$ can potentially mix with $\not h \phi$. In the continuum, this mixing is excluded by chiral symmetry, and thus $\phi$ has a simple multiplicative renormalization. However, commonly used lattice regularizations break chiral symmetry, and therefore mixing of these two operators must be accounted for in the renormalization procedure. We thus obtain the renormalization pattern

$$
\phi_{R}=Z_{\phi}\left(\phi+r_{\text {mix }} h \phi\right), \quad \bar{\phi}_{R}=Z_{\phi}\left(\bar{\phi}+r_{\text {mix }} \bar{\phi} \not h\right) .
$$

We can also use projectors to form operators $\phi^{ \pm} \equiv \frac{1}{2}(1 \pm \not h) \phi$ that renormalize diagonally with $Z_{\phi}^{ \pm} \equiv Z_{\phi}\left(1 \pm r_{\text {mix }}\right)$. This pattern leads to the form of the renormalized $\mathcal{O}_{\Gamma}$, for $\xi \neq 0$ :

$$
\begin{aligned}
\mathcal{O}_{\Gamma}^{R}(x, \xi, n) & =Z_{\phi}^{2} e^{-m|\xi|} \mathcal{O}_{\Gamma^{\prime}}(x, \xi, n), \\
\Gamma^{\prime} & =\Gamma+r_{\text {mix }} \operatorname{sgn}(\xi)\{\not h, \Gamma\}+r_{\text {mix }}^{2} \not h \not h .
\end{aligned}
$$

$\mathcal{O}_{\Gamma}$ can therefore be renormalized by determining three parameters: the linearly divergent $m$, the $\log$-divergent $Z_{\phi}$, and the finite $r_{\text {mix }}$. Since $r_{\text {mix }}$ is $O\left(g^{2}\right)$, this is the same pattern at one-loop order as in Ref. [14]. At $\xi=0, \mathcal{O}_{\Gamma}$ is a local quark bilinear with a different divergence structure and should have a separate renormalization factor [20], which can be computed in the usual way; we use results from Ref. [21]. We also note that, since the local operator $\phi$ is not flavor singlet, there is no mixing between quark and gluon quasi-PDFs even when $\mathcal{O}_{\Gamma}$ is flavor singlet. In the latter case, mixing will occur in the matching to PDFs.

If we choose $n=\hat{t}$ and give $\zeta$ spin degrees of freedom (which do not couple in the action), then $S_{\zeta}$ becomes the action for a static quark on the lattice [22,23]. In particular, $\phi$ is related to the static-light bilinears, and we find relations between renormalization factors: $Z_{V}^{\text {stat }}=Z_{\phi}^{+}$ and $Z_{A}^{\text {stat }}=Z_{\phi}^{-}$. The static quark theory also tells us how to remove $O(a)$ lattice artifacts [24], which are present even if chiral symmetry is preserved on the lattice. In the continuum, the relation between $\mathcal{O}_{\Gamma}$ and the static quark theory was previously discussed in Ref. [25].

We determine the renormalization parameters nonperturbatively using a variant of the Rome-Southampton method. In the Landau gauge on $N_{f}=4$ twisted mass lattice ensembles [21], we compute the position-space $\zeta$ propagator

$S_{\zeta}(\xi) \equiv\langle\zeta(x+\xi n) \bar{\zeta}(x)\rangle_{\mathrm{QCD}+\zeta}=\langle W(x+\xi n, x)\rangle_{\mathrm{QCD}}$

the momentum-space quark propagator $S_{\psi}(p)$, and the mixed-space Green's function for $\phi^{ \pm}$:

$$
G^{ \pm}(\xi, p) \equiv \int d^{4} x e^{i p \cdot x}\left\langle\zeta(\xi n) \phi^{ \pm}(0) \bar{\psi}(x)\right\rangle_{\mathrm{QCD}+\zeta} \cdot
$$

Similarly to the approach used for quark bilinears [17], the renormalization parameters as well as the $\zeta$ and $\psi$ field renormalizations can be determined by imposing conditions that require an appropriately chosen set of renormalized correlators to equal their tree-level values. Specifically, for kinematics given by the momentum $p_{0}$ and distance $\xi_{0}$, we require

$$
-\left.\frac{d}{d \xi} \log \operatorname{Tr} S_{\zeta}(\xi)\right|_{\xi=\xi_{0}}+m=0
$$




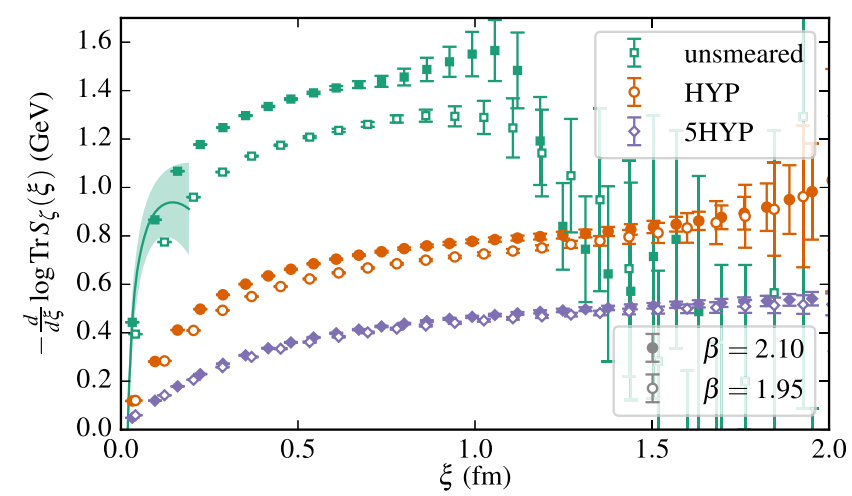

FIG. 1. Effective energy of the bare auxiliary field propagator, for two lattice spacings and three different link discretizations. Solid symbols show the finer lattice spacing, and open symbols show the coarser one. The curve shows the three-loop perturbative result, shifted vertically by $-m$ to match it to the unsmeared data on the finer lattice spacing. Its error band indicates the size of the $O\left(\alpha_{s}^{3}\right)$ contribution.

$$
\begin{gathered}
\frac{Z_{\zeta}}{3} \frac{\left[\operatorname{Tr} S_{\zeta}\left(\xi_{0}\right)\right]^{2}}{\operatorname{Tr} S_{\zeta}\left(2 \xi_{0}\right)}=1, \\
\frac{1}{6} \frac{Z_{\phi}^{ \pm}}{\sqrt{Z_{\zeta} Z_{\psi}}} \operatorname{Re} \operatorname{Tr}\left[S_{\zeta}^{-1}\left(\xi_{0}\right) G^{ \pm}\left(\xi_{0}, p_{0}\right) S_{\psi}^{-1}\left(p_{0}\right)\right]=1
\end{gathered}
$$

and use the same condition for $S_{\psi}$ as in the RI'-MOM and symmetric RI-SMOM schemes [17,26]. The linearly divergent counterterm $m$ can be determined using Eq. (9), whereas the other conditions are constructed to eliminate dependence on it, so that the other renormalization parameters can be determined independently of $m$. Rewriting the parameters $\left(p_{0}, \xi_{0}\right)$, these conditions define a two-parameter family of renormalization schemes at scale $\mu^{2}=p_{0}^{2}$ that depend on the dimensionless quantities $y \equiv\left|p_{0}\right| \xi_{0}$ and $z \equiv p_{0} \cdot n /\left|p_{0}\right|$. We call this family of schemes RI-xMOM. Restricting to the kinematics $p_{0} \propto n$ (i.e., $z=1$ ), we have computed the conversion to the $\overline{\mathrm{MS}}$ scheme at one-loop order, using dimensionally regularized perturbation theory [27]. We obtain in the Landau gauge

$$
\begin{aligned}
C_{\phi} \equiv & \frac{Z_{\phi}^{\overline{\mathrm{MS}}}(\mu)}{Z_{\phi}^{\mathrm{RI}-\mathrm{xMOM}}(\mu, y, z=1)} \\
= & 1+\frac{\alpha_{s} C_{F}}{8 \pi}\left[6 \log \frac{y}{4}+6 \gamma_{E}-8 \log 2+7-\cos y\right. \\
& \left.-\left(8 \cos \frac{y}{2}-y \sin \frac{y}{2}\right) \operatorname{Ci}\left(\frac{y}{2}\right)+8 \operatorname{Ci}(y)\right]+O\left(\alpha_{s}^{2}\right),
\end{aligned}
$$

where $\gamma_{E}$ is the Euler-Mascheroni constant and $\mathrm{Ci}$ is the cosine integral function, $\operatorname{Ci}(z) \equiv-\int_{z}^{\infty} t^{-1} \cos (t) d t$. For

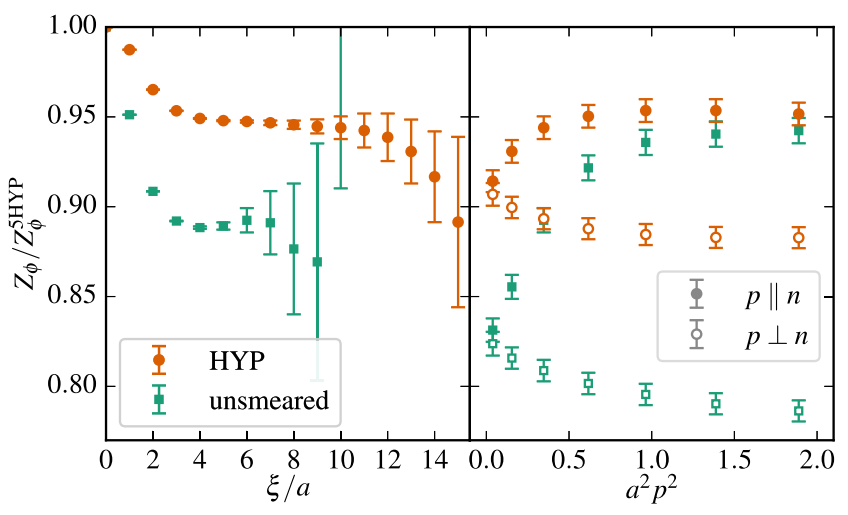

FIG. 2. $Z_{\phi}$ for $\beta=2.10$, relative to the 5HYP case. Left: Versus $\xi$, for $p \| n$ and $a^{2} p^{2} \approx 0.35$. Right: Versus $p^{2}$, for $\xi=6 a$ (unsmeared) and $\xi=10 a$ (HYP). For each link discretization, we take the average of the two values at the smallest $p^{2}$.

converting $m$ to the $\overline{\mathrm{MS}}$ scheme, we use the three-loop results for the static quark propagator from Refs. [28,29].

In Fig. 1, we show the quantity in Eq. (9), for two different lattice spacings: $a=0.082 \mathrm{fm}(\beta=1.95)$ and $a=0.064 \mathrm{fm}(\beta=2.10)$. (This is the "effective energy" of the auxiliary field propagator. It was previously studied in Ref. [30], where it was denoted $Y_{\text {line. }}$ ) It is renormalized by adding $m$. Without smearing, there is a significant difference between the two lattice spacings due to the linear divergence, but this is greatly reduced by applying one or five steps of hypercubic (HYP) smearing [31], which also reduces the statistical uncertainty at large $\xi$. At small $\xi / a$, smearing distorts the shape, and therefore we choose to impose our condition at $\xi_{0} \approx 0.6 \mathrm{fm}$, where the shapes are similar. We then convert to the $\overline{\mathrm{MS}}$ scheme at a short

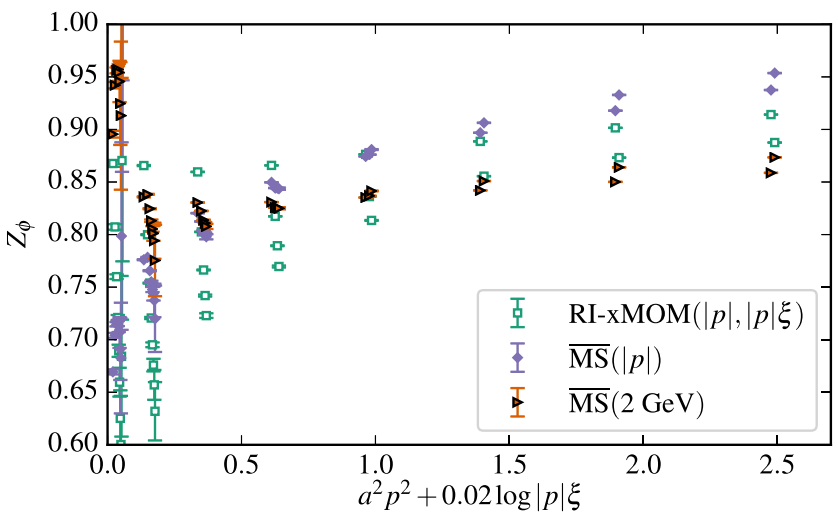

FIG. 3. $Z_{\phi}$ for $\beta=2.10$, using unsmeared gauge links. Data are shown for a range of $p^{2}$ and $y \equiv|p| \xi$; the horizontal axis is $a^{2} p^{2}$, with a small displacement for different $y$ at the same $p^{2}$. The green open squares are given in our family of schemes, the blue solid diamonds show the result from conversion to $\overline{\mathrm{MS}}$ at scale $|p|$ using Eq. (12), and the orange solid triangles with black outlines show the $\overline{\mathrm{MS}}$ results evolved to the scale $2 \mathrm{GeV}$, using the twoloop anomalous dimension of the static-light current [28,34,35]. 

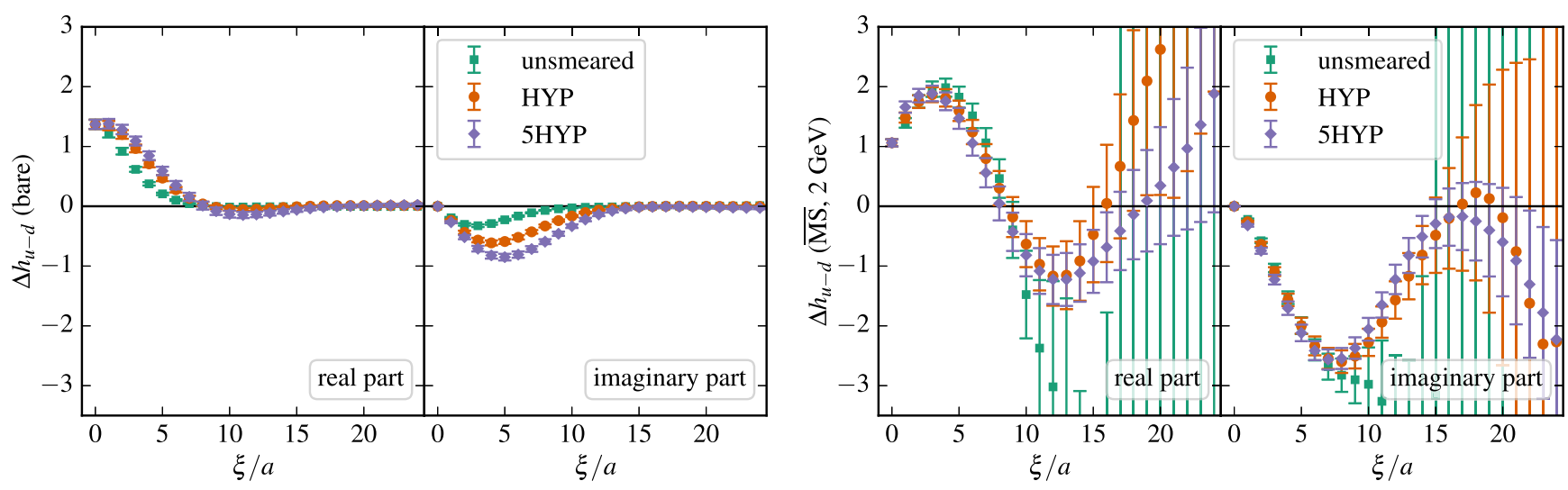

FIG. 4. Matrix element for the helicity quasi-PDF versus $\xi$ on the $\beta=2.10$ ensemble, for three different link discretizations, bare (left) and renormalized (right). Only $\xi \geq 0$ is shown, since the real part is even in $\xi$ and the imaginary part is odd.

distance by matching the result using unsmeared links on the fine ensemble to the perturbative result.

From Eq. (11), an estimator for $r_{\text {mix }}$ can be isolated. In our data, we see indications that this suffers from significant $O(a)$ lattice artifacts. These could be remedied through Symanzik improvement [24], but instead we choose to focus on the helicity quasi-PDF, which is unaffected by mixing and depends only on $r_{\text {mix }}^{2}$ [32]. We find that the latter is not much greater than $1 \%$ and can be neglected at the current level of precision.

The remaining factor $Z_{\phi}$ depends on the kinematics that define our scheme, but the ratio between $Z_{\phi}$ for different discretizations is scheme independent. We evaluate this ratio in the plateau region at large $\xi$ and at small $p$ (Fig. 2) to reduce lattice artifacts. Finally, we determine $Z_{\phi}$ using unsmeared gauge links, converting to the $\overline{\mathrm{MS}}$ scheme and evolving to the scale $2 \mathrm{GeV}$, as shown in Fig. 3. We find that the one-loop conversion factor is effective at removing much of the dependence on the scheme parameter $|p| \xi$, and the two-loop evolution removes most of the dependence on the scale $|p|$.

We apply these renormalization parameters to nucleon matrix elements computed on one $N_{f}=2+1+1$ twisted mass ensemble at each lattice spacing. The physical parameters are matched on the two ensembles: $m_{\pi} \approx$ $370 \mathrm{MeV}$ and $p \cdot n \approx 1.85 \mathrm{GeV}$. The coarser ensemble was previously used in Refs. [3,13], and our methodology is similar to Ref. [13], including the use of momentum smearing [36] in the nucleon interpolating operator to obtain a good signal at large momentum.

Figure 4 shows the effect of renormalization on the isovector helicity matrix element $\Delta h_{u-d}$,

$$
\left\langle p, \lambda^{\prime}\left|\mathcal{O}_{\mu \gamma_{5}}\right| p, \lambda\right\rangle \equiv \bar{u}\left(p, \lambda^{\prime}\right) \not h \gamma_{5} u(p, \lambda) \Delta h(\xi, p \cdot n)
$$

computed on the fine ensemble, for different link discretizations. Without renormalization, there is a significant disagreement between the different link types, and renormalization brings them into good agreement. In the renormalized matrix elements, we also see the benefit of smearing: without it, the statistical errors grow rapidly at large $\xi$ and there is no useful signal for $\xi \gtrsim 10 a$. With smearing, we are able to see that the matrix elements return toward zero at large $\xi$. Five steps of HYP smearing also yield more precise data than one step, at large $\xi$. In Fig. 5, we compare the renormalized data with five steps of HYP smearing on the two ensembles. They are in excellent agreement, which suggests that the linear divergence is under control and discretization effects are not large.

Finally, we examine the helicity quasi-PDF, which is given by a Fourier transform of the matrix element:

$$
\Delta \tilde{q}(x, p \cdot n) \equiv \frac{p \cdot n}{2 \pi} \int d \xi e^{-i x \xi p \cdot n} \Delta h_{q}(\xi, p \cdot n) .
$$

Without renormalization, unsmeared links lead to a much broader distribution, as shown in Fig. 4 of Ref. [3]. We show our renormalized results on the fine ensemble in Fig. 6. Because the data become noisy at large $\xi$, we restrict the integral to $|\xi| \leq 16 a(|\xi| \leq 10 a$ for the unsmeared case).

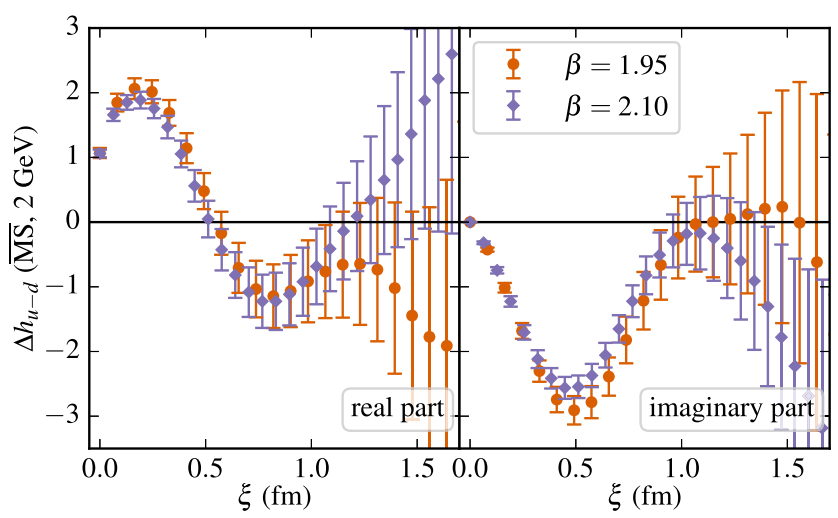

FIG. 5. Renormalized matrix element for the helicity quasiPDF versus $\xi$ on the two ensembles, using five steps of HYP smearing. 


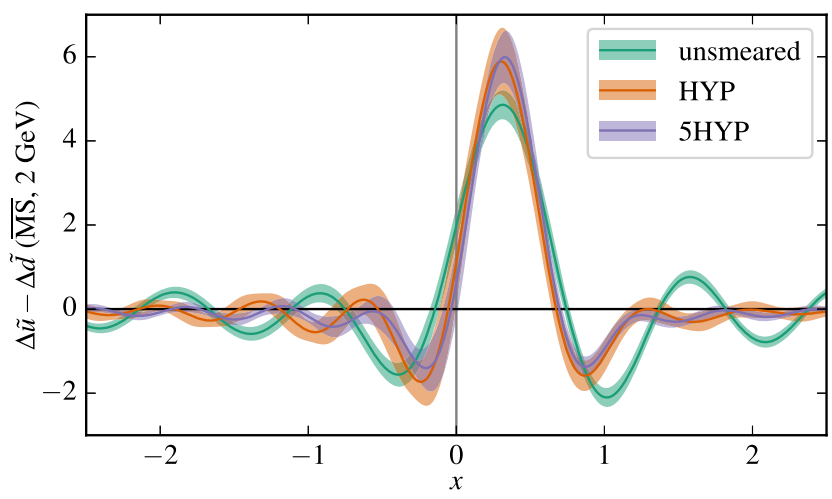

FIG. 6. Isovector helicity quasi-PDF on the $\beta=2.10$ ensemble, for three different link discretizations, computed from renormalized matrix elements.

When this restriction removes part of the signal, it leads to oscillations, which are clearly visible in the unsmeared case. In future studies, improved results could be obtained by replacing the hard cutoff with a model for the large- $\xi$ behavior of the matrix elements or by applying one of the methods proposed in Refs. [37,38] for suppressing the contributions at large $\xi$. Ignoring the oscillations, we see that renormalization brings the data with different link discretizations into reasonably good agreement.

In this work, we have shown that the nonlocal problem of renormalizing lattice quasi-PDFs can be turned into a local problem by introducing an auxiliary field. The auxiliary field approach can also be applied to operators with staple-shaped gauge connection used for lattice studies of transverse momentum-dependent PDFs [8-10], where the mixing pattern will be different. Because this approach is closely connected with the static quark theory, it is possible to make use of existing results from that theory such as the three-loop continuum calculation in Ref. [28]. We have contributed to the evidence that link smearing is a very beneficial technique for these calculations, as it leads to a greatly reduced uncertainty at large $\xi$; this was also explained some time ago in the static quark theory [39]. There are several steps from the results shown here to a full calculation of PDFs: matching from quasi-PDFs to PDFs, control over the $p \cdot n \rightarrow$ $\infty$ limit, and use of a physical pion mass, as well as control over finite-volume and excited-state effects.

We thank our colleagues in the European Twisted Mass Collaboration (ETMC) for a pleasant collaboration. Gauge fixing was performed using the Fourier-accelerated conjugate gradient [40], implemented in Gauge Link Utility (GLU) [41]. Calculations were performed using the Grid library [42] and the DD- $\alpha$ AMG solver [43] with twisted mass support [44]. The authors gratefully acknowledge the computing time granted by the John von Neumann Institute for Computing (NIC) and provided on the supercomputer JURECA [45] at Jülich Supercomputing Centre (JSC). F. S. was funded by Deutsche Forschungsgemeinschaft (DFG) Project No. 392578569.
Note added.-After one of us presented a preliminary version of this work at a conference [46], we were made aware of an independent parallel effort based on the auxiliary field approach [47]. We also note another very recent article discussing renormalizability of quasi-PDFs, without using the auxiliary field approach [48].

*jeremy.green@desy.de

[1] X. Ji, Parton Physics on a Euclidean Lattice, Phys. Rev. Lett. 110, 262002 (2013).

[2] X. Xiong, X. Ji, J.-H. Zhang, and Y. Zhao, One-loop matching for parton distributions: Nonsinglet case, Phys. Rev. D 90, 014051 (2014).

[3] C. Alexandrou, K. Cichy, V. Drach, E. Garcia-Ramos, K. Hadjiyiannakou, K. Jansen, F. Steffens, and C. Wiese, Lattice calculation of parton distributions, Phys. Rev. D 92, 014502 (2015).

[4] T. Ishikawa, Y.-Q. Ma, J.-W. Qiu, and S. Yoshida, Practical quasi parton distribution functions, arXiv:1609.02018.

[5] J.-W. Chen, X. Ji, and J.-H. Zhang, Improved quasi parton distribution through Wilson line renormalization, Nucl. Phys. B915, 1 (2017).

[6] A. V. Radyushkin, Quasi-parton distribution functions, momentum distributions, and pseudo-parton distribution functions, Phys. Rev. D 96, 034025 (2017).

[7] K. Orginos, A. Radyushkin, J. Karpie, and S. Zafeiropoulos, Lattice QCD exploration of parton pseudo-distribution functions, Phys. Rev. D 96, 094503 (2017).

[8] B. U. Musch, P. Hägler, M. Engelhardt, J. W. Negele, and A. Schäfer, Sivers and Boer-Mulders observables from lattice QCD, Phys. Rev. D 85, 094510 (2012).

[9] M. Engelhardt, P. Hägler, B. Musch, J. Negele, and A. Schäfer, Lattice QCD study of the Boer-Mulders effect in a pion, Phys. Rev. D 93, 054501 (2016).

[10] B. Yoon, M. Engelhardt, R. Gupta, T. Bhattacharya, J. R. Green, B. U. Musch, J. W. Negele, A. V. Pochinsky, A. Schäfer, and S. N. Syritsyn, Nucleon transverse momentumdependent parton distributions in lattice QCD: Renormalization patterns and discretization effects, Phys. Rev. D 96, 094508 (2017)

[11] H.-W. Lin, J.-W. Chen, S. D. Cohen, and X. Ji, Flavor structure of the nucleon sea from lattice QCD, Phys. Rev. D 91, 054510 (2015).

[12] J.-W. Chen, S. D. Cohen, X. Ji, H.-W. Lin, and J.-H. Zhang, Nucleon helicity and transversity parton distributions from lattice QCD, Nucl. Phys. B911, 246 (2016).

[13] C. Alexandrou, K. Cichy, M. Constantinou, K. Hadjiyiannakou, K. Jansen, F. Steffens, and C. Wiese, Updated lattice results for parton distributions, Phys. Rev. D 96, 014513 (2017).

[14] M. Constantinou and H. Panagopoulos, Perturbative renormalization of quasi-parton distribution functions, Phys. Rev. D 96, 054506 (2017).

[15] C. Alexandrou, K. Cichy, M. Constantinou, K. Hadjiyiannakou, K. Jansen, H. Panagopoulos, and F. Steffens, A complete non-perturbative renormalization prescription for quasi-PDFs, Nucl. Phys. B923, 394 (2017). 
[16] J.-W. Chen, T. Ishikawa, L. Jin, H.-W. Lin, Y.-B. Yang, J.-H. Zhang, and Y. Zhao, Parton distribution function with nonperturbative renormalization from lattice QCD, Phys. Rev. D 97, 014505 (2018).

[17] G. Martinelli, C. Pittori, C. T. Sachrajda, M. Testa, and A. Vladikas, A general method for nonperturbative renormalization of lattice operators, Nucl. Phys. B445, 81 (1995).

[18] N.S. Craigie and H. Dorn, On the renormalization and short distance properties of hadronic operators in QCD, Nucl. Phys. B185, 204 (1981).

[19] H. Dorn, Renormalization of path ordered phase factors and related hadron operators in gauge field theories, Fortschr. Phys. 34, 11 (1986).

[20] M. Constantinou (private communication).

[21] C. Alexandrou, M. Constantinou, and H. Panagopoulos (ETM Collaboration), Renormalization functions for $N_{f}=2$ and $N_{f}=4$ twisted mass fermions, Phys. Rev. D 95, 034505 (2017).

[22] E. Eichten and B. R. Hill, An effective field theory for the calculation of matrix elements involving heavy quarks, Phys. Lett. B 234, 511 (1990).

[23] R. Sommer, in Modern Perspectives in Lattice QCD: Quantum Field Theory and High Performance Computing. Proceedings, International School, 93rd Session, Les Houches, France, 2009 (Oxford University Press, New York, 2010), pp. 517-590.

[24] M. Kurth and R. Sommer (ALPHA Collaboration), Renormalization and $O(a)$ improvement of the static axial current, Nucl. Phys. B597, 488 (2001).

[25] X. Ji and J.-H. Zhang, Renormalization of quasiparton distribution, Phys. Rev. D 92, 034006 (2015).

[26] C. Sturm, Y. Aoki, N. H. Christ, T. Izubuchi, C. T. C. Sachrajda, and A. Soni, Renormalization of quark bilinear operators in a momentum-subtraction scheme with a nonexceptional subtraction point, Phys. Rev. D 80, 014501 (2009).

[27] J. Green, K. Jansen, and F. Steffens (to be published).

[28] K. G. Chetyrkin and A. G. Grozin, Three loop anomalous dimension of the heavy light quark current in HQET, Nucl. Phys. B666, 289 (2003).

[29] K. Melnikov and T. van Ritbergen, The three loop on-shell renormalization of QCD and QED, Nucl. Phys. B591, 515 (2000).

[30] B. U. Musch, P. Hägler, J. W. Negele, and A. Schäfer, Exploring quark transverse momentum distributions with lattice QCD, Phys. Rev. D 83, 094507 (2011).

[31] A. Hasenfratz and F. Knechtli, Flavor symmetry and the static potential with hypercubic blocking, Phys. Rev. D 64, 034504 (2001).

[32] Following the same logic used to derive automatic $O(a)$ improvement [33], it can also be argued that, for twisted mass lattice QCD at maximal twist, the contribution from the mixing operator to nucleon matrix elements will vanish at $O(a)$.

[33] R. Frezzotti and G. C. Rossi, Chirally improving Wilson fermions 1. $O(a)$ improvement, J. High Energy Phys. 08 (2004) 007.

[34] X. Ji and M.J. Musolf, Sub-leading logarithmic massdependence in heavy-meson form-factors, Phys. Lett. B 257, 409 (1991).

[35] D. J. Broadhurst and A. G. Grozin, Two-loop renormalization of the effective field theory of a static quark, Phys. Lett. B 267, 105 (1991).

[36] G. S. Bali, B. Lang, B. U. Musch, and A. Schäfer, Novel quark smearing for hadrons with high momenta in lattice QCD, Phys. Rev. D 93, 094515 (2016).

[37] H.-W. Lin, J.-W. Chen, T. Ishikawa, and J.-H. Zhang, Improved parton distribution functions at physical pion mass, arXiv:1708.05301.

[38] J.-W. Chen, T. Ishikawa, L. Jin, H.-W. Lin, A. Schäfer, Y.-B. Yang, J.-H. Zhang, and Y. Zhao, Gaussian-weighted parton quasi-distribution, arXiv:1711.07858.

[39] M. Della Morte, S. Dürr, J. Heitger, H. Molke, J. Rolf, A. Shindler, and R. Sommer (ALPHA Collaboration), Lattice HQET with exponentially improved statistical precision, Phys. Lett. B 581, 93 (2004); Erratum 612, 313 (2005).

[40] R. J. Hudspith (RBC, UKQCD Collaboration), Fourier accelerated conjugate gradient lattice gauge fixing, Comput. Phys. Commun. 187, 115 (2015).

[41] R. J. Hudspith, Gauge link utility, https://github.com/ RJhudspith/GLU.

[42] P. A. Boyle, G. Cossu, A. Yamaguchi, and A. Portelli, Grid: A next generation data parallel C++ QCD library, Proc. Sci., LATTICE2015 (2016) 023 [arXiv:1512.03487].

[43] A. Frommer, K. Kahl, S. Krieg, B. Leder, and M. Rottmann, Adaptive aggregation-based domain decomposition multigrid for the lattice Wilson-Dirac operator, SIAM J. Sci. Comput. 36, A1581 (2014).

[44] C. Alexandrou, S. Bacchio, J. Finkenrath, A. Frommer, K. Kahl, and M. Rottmann, Adaptive aggregation-based domain decomposition multigrid for twisted mass fermions, Phys. Rev. D 94, 114509 (2016).

[45] Jülich Supercomputing Centre, JURECA: General-purpose supercomputer at Jülich Supercomputing Centre, J. LargeScale Res. Facilities 2, A62 (2016).

[46] J. Green, in 35th International Symposium on Lattice Field Theory (Lattice 2017), Granada, Spain, 2017 (unpublished), https://makondo.ugr.es/event/0/session/95/contribution/332.

[47] X. Ji, J.-H. Zhang, and Y. Zhao, Renormalization in Large Momentum Effective Theory of Parton Physics, Phys. Rev. Lett. 120, 112001 (2018).

[48] T. Ishikawa, Y.-Q. Ma, J.-W. Qiu, and S. Yoshida, Renormalizability of quasiparton distribution functions, Phys. Rev. D 96, 094019 (2017). 